\title{
Triple-Resonant Brillouin Light Scattering in Magneto-Optical Cavities
}

\author{
J. A. Haigh, ${ }^{1, *}$ A. Nunnenkamp, ${ }^{2}$ A. J. Ramsay, ${ }^{1}$ and A. J. Ferguson ${ }^{2}$ \\ ${ }^{1}$ Hitachi Cambridge Laboratory, Cambridge CB3 OHE, United Kingdom \\ ${ }^{2}$ Cavendish Laboratory, University of Cambridge, Cambridge CB3 OHE, United Kingdom
}

(Received 11 July 2016; published 21 September 2016)

\begin{abstract}
An enhancement in Brillouin light scattering of optical photons with magnons is demonstrated in magneto-optical whispering gallery mode resonators tuned to a triple-resonance point. This occurs when both the input and output optical modes are resonant with those of the whispering gallery resonator, with a separation given by the ferromagnetic resonance frequency. The identification and excitation of specific optical modes allows us to gain a clear understanding of the mode-matching conditions. A selection rule due to wave vector matching leads to an intrinsic single-sideband excitation. Strong suppression of one sideband is essential for one-to-one frequency mapping in coherent optical-to-microwave conversion.
\end{abstract}

DOI: 10.1103/PhysRevLett.117.133602

Extending microwave-optical transducers into a regime where interconversion between single optical and microwave photons is possible in a coherent manner [1] is an important technological aim, as it would open up many avenues in, for example, implementing existing superconducting quantum devices [2] in a wider quantum network [3]. Furthermore, frequency shifting of single photons would enable quantum optical devices to take advantage of wavelength division multiplexing. Strong progress towards these goals has been made in cavity optomechanics [4-7], and optimized electro-optic modulators [8-10].

Recently, microwave-optical interconversion has also been explored in a cavity optomagnonic system [11,12], where magnetic Brillouin light scattering (BLS) [13] has been reported in high $Q$ optical whispering gallery modes (WGMs) of a transparent magnetic sphere [14]. In this system, the collective excitations of the magnetic moment, magnons, play a role analogous to the phonons in a cavity optomechanics system [15]. An important feature of this optomagnonic system is the nonreciprocity of the BLS, where only one sideband has been observed [11,12]. A key requirement for a coherent transducer is a one-to-one mapping of the frequency components, and hence a strong suppression of one sideband. In contrast to an optomechanics system, due to conservation of angular momentum, optically induced creation and annihilation of magnons requires a change in optical polarization [16]. When combined with the geometric birefringence of a WGM resonator, this results in a nonreciprocal triple-resonance condition, where the optical pump and signal of opposite polarization are resonant with different cavity modes, whose frequency splitting is equal to the driven ferromagnetic resonance (FMR) [17]. Hence, sideband suppression is enforced by a selection rule, rather than by detuning the pump laser from the optical cavity, as is usually the case in a cavity-optomechanics system.

In this Letter we show that the nonreciprocal tripleresonance condition between optical modes for pump and signal of the interconversion can be achieved with the precise mode identification allowed by prism coupling to the magnetic sphere. This is in contrast to previous measurements [11,12], where, due to the waveguide coupling used, the exact identification of the optical modes involved has been difficult, with the resonance condition being met accidentally [12]. For microwave driving of the uniform Kittel magnetization mode in the plane of the WGM, the polarization of the pump laser can be used to select the scattering direction via the fixed change in the azimuthal mode index. We identify that this selectivity arises from wave vector matching around the optical path of the pump and signal light fields and the geometrical dependence of the magneto-optical coupling. Finally, measurements of the BLS intensity as a function of detuning from the triple-resonance condition show excellent agreement with a simple analytical model. Our experiments allow us to precisely characterize the resonant singlephoton magneto-optical coupling strength [18,19].

The experimental setup is shown in Fig. 1(a). A prism coupler [20] is used to match the input angle, and therefore the wave vector, to the low order WGMs. The mode structure is probed by measuring the reflected intensity with the same polarization as the input using a photodiode (PD I), as the input laser wavelength is tuned. The light emitted from the cavity with opposite linear polarization to the input is emitted at a different angle due to the birefringence of the rutile prism. This polarization-scattered component is analyzed with a scanning Fabry-Pérot etalon on an avalanche photodiode (PD II). A microwave antenna [Fig. 1(b)] is placed close to the YIG sphere to drive ferromagnetic resonance and the magnetic field from a permanent magnet $(\mathrm{NdFeB})$ mounted on a stage is used to tune the FMR frequency. The setup can be switched to measure the same quantities for both linear polarizations of the input beam.

First, we identify the optical WGMs. The dc magnetic field is fixed in the out-of-plane direction. Since there is no 


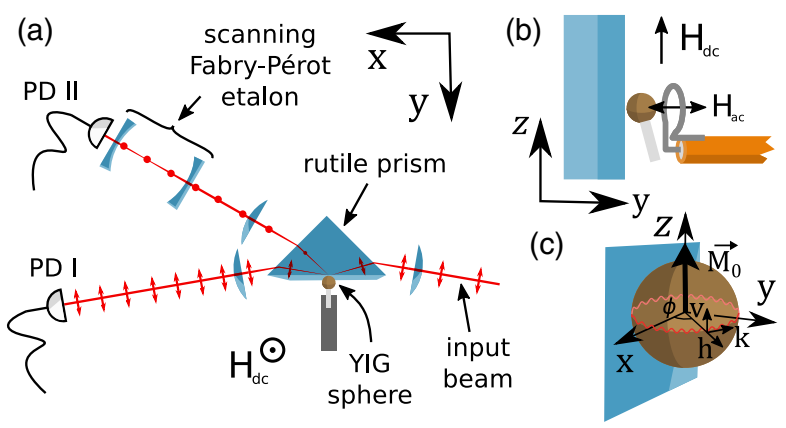

FIG. 1. (a) Top view of experimental setup. The scattered light, with polarization orthogonal to the input beam, emitted at a different angle due to the birefringence of the rutile coupling prism, is spectroscopically analyzed with a scanning Fabry-Pérot etalon. A dc magnetic field $H_{\mathrm{dc}}$ is applied along the $z$ axis. (b) Microwave antenna to drive ferromagnetic resonance in the YIG sphere (side view). The microwave drive is provided by a vector network analyzer (VNA). The FMR modes are identified by measuring the microwave reflection coefficient $\left|S_{11}\right|$ as a function of frequency, after which the VNA is configured in the continuous-wave mode to drive the resonance. (c) Coordinate systems used in the analysis.

static component of the magnetization along the direction of propagation, mixing between linear polarized modes due to the Faraday effect is negligible [14]. We therefore use the standard analytical forms of the WGM electric field distributions and resonant wavelengths [21,22], with two linearly polarized components perpendicular and parallel to the sphere surface. These modes, which we label horizontal $h$ and vertical $v$ [23] [see Fig. 1(c)], are split in energy due to the geometrical birefringence from the different surface boundary conditions for two electric field components.

The basic mode structure is shown schematically in Fig. 2(a). The expected reflectance spectra for the $h$ (pink) and $v$ (green) polarized modes are shown including modes with radial index $q=1,2$ for sets of modes with a difference $\Delta m=1$ in the azimuthal index $m$. The mode indices are defined in Fig. 2(b). The free spectral range is given by $\lambda_{\mathrm{FSR}}=\lambda_{0}^{2} / 2 \pi r n_{\mathrm{YIG}}$ to a good approximation in the relatively large spheres $(r \sim 100 \mu \mathrm{m}, m \sim 1000)$ which we study. In the same limit, the $h-v$ splitting is given by $\lambda_{h-v}=\lambda_{\mathrm{FSR}} \sqrt{n_{\mathrm{YIG}}^{2}-1} / n_{\mathrm{YIG}}$ [21]. For YIG, with $n_{\mathrm{YIG}} \approx 2.2$, $\lambda_{h-v} \approx 0.9 \lambda_{\mathrm{FSR}}$. Therefore, the closest adjacent modes of opposite polarization are for different $m$ indices, separated by $m_{v}-m_{h}=1$ and $\lambda_{h-v}^{\text {eff }}=0.1 \lambda_{\mathrm{FSR}}$.

Figure 2(c) presents a reflection spectrum for an $h$ polarized input. Two families of modes are observed. These are identified as $q=1$ and $q=2$ from comparison of the wavelength dispersion, shown in Fig. 2(d), to the expected splitting. This demonstrates the highly selective excitation of the WGM, allowing clear identification of the matching conditions for enhanced wavelength conversion.

With the dc magnetic field in the out-of-plane direction $z$, we now introduce the microwave drive field in the in-plane
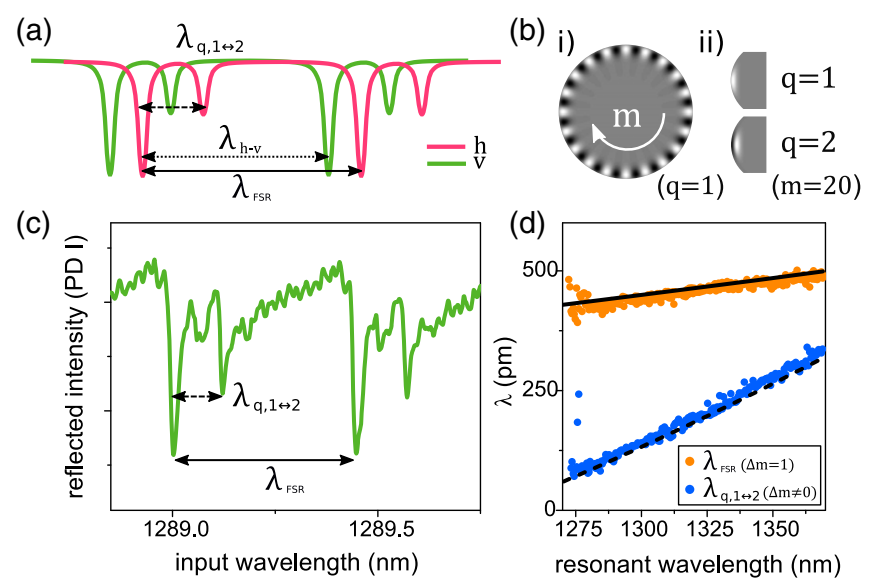

FIG. 2. Optical mode identification for an $r \approx 250 \mu \mathrm{m}$ YIG sphere. (a) Schematic of mode families for radial indices $q=1$, 2 , and for $h$ and $v$ polarization. The free spectral range $\lambda_{\mathrm{FSR}}$ is indicated by the black solid line. The $h-v$ splitting $\lambda_{h-v}$ is shown by the black dotted line, while the dashed line indicates the splitting between adjacent modes with $\Delta q=1$. The azimuthal index $m$ determines the number of wavelengths around the circumference and radial index $q$ determines the number of radial nodes. (b) Representative plots of the real part of the electric field (i) in the WGM plane for $m=20, q=1$, and (ii) in cross section for $q=1,2$. (c) Reflectance spectrum for $v$-polarized input. (d) The dispersion of the FSR $\lambda_{\mathrm{FSR}}$ is used to identify the strongest mode family as $q=1$. The splitting between the different modes $\lambda_{q, 1 \leftrightarrow 2}$ is used to identify the second as $q=2$. Solid and dashed lines show the calculated dispersions [24] fitted with small adjustments of the sphere radius.

$x$ direction. This drives ferromagnetic resonance (FMR), the precession of the magnetization about the static field. The magnetostatic modes [25] of the YIG sphere can be identified by measuring the microwave reflection coefficient $S_{11}$ of the microwave antenna. The FMR spectrum as a function of permanent magnet position is shown in Fig. 3(a) along with the expected Kittel mode frequency calculated from the position dependent magnetic field (blue line) [26]. From this field dependence and the relative strength of the absorption, the uniform Kittel mode can be identified. During data collection the microwave drive tracks the FMR frequency to compensate for fluctuations in the dc magnetic field.

To achieve the triple-resonance condition, we use a sphere of radius $500 \mu \mathrm{m}$, which has $\lambda_{h-v}^{\text {eff }}$ corresponding to $\omega_{v}-\omega_{h} \approx 7 \mathrm{GHz}$, and drive the FMR of the uniform Kittel mode close to that frequency. The cross-polarized emission of the cavity is spectrally analyzed using the etalon, and example data are shown in Fig. 3(b). The top panel shows a measured spectrum for $h$ polarized input. There are two sets of peaks, each matching the $10 \mathrm{GHz}$ FSR of the etalon. The largest is the elastic scattered light at the same wavelength as the input laser. The anti-Stokes signal is marked with a blue arrow and is higher in frequency by $\approx 7 \mathrm{GHz}$. There is no measurable Stokes peak for this input 

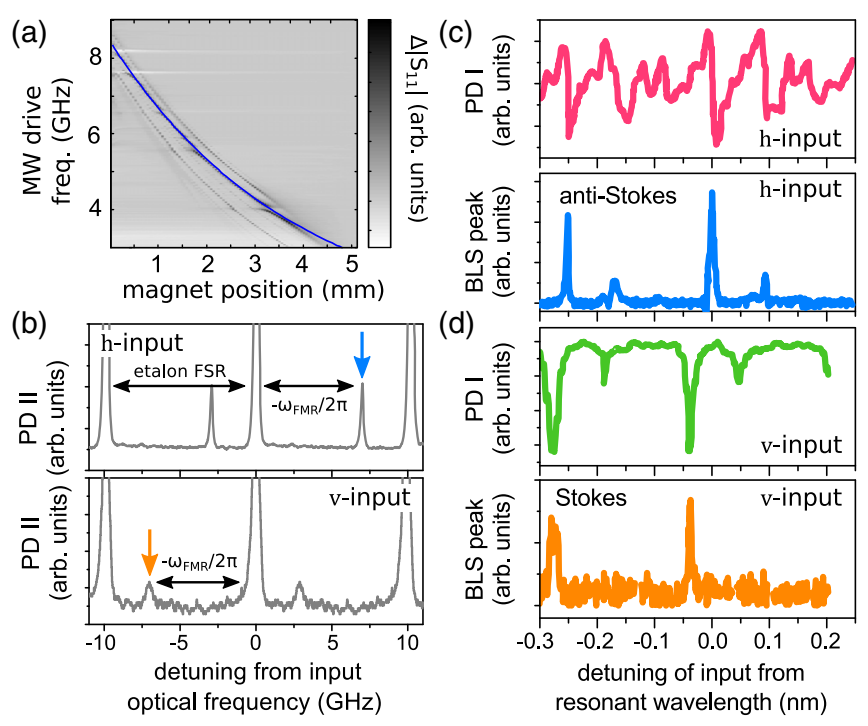

FIG. 3. (a) FMR of YIG sphere measured through microwave reflection coefficient $\left|S_{11}\right|$ of the antenna as a function of permanent magnet position. The blue dashed line shows the expected dependence of the uniform Kittel mode from the known dependence of the magnetic field on distance from a cuboid magnet [26] and the gyromagnetic ratio $\gamma=28 \mathrm{GHz} \mathrm{T}^{-1}$. The magnetic field range is $H_{\mathrm{dc}} \approx 100-320 \mathrm{mT}$. The $Q$ factor of the magnetic mode is $Q_{\mathrm{FMR}} \approx 400$ (as this is due to Gilbert damping, the rate $\kappa_{\mathrm{FMR}} \approx 10-20 \mathrm{MHz}$ depends linearly on FMR frequency [27]). (b) Measured spectra of emitted signal (PD II) for $h$ (upper) an $v$ input polarization for $\omega_{\mathrm{FMR}} / 2 \pi \approx 7 \mathrm{GHz}$. Orange and blue arrows label Stokes and anti-Stokes peaks, respectively. We can exclude the suppressed sideband down to the signal-to-noise ratio, maximum $\approx 20$ (slightly different for the two input polarizations due to different experimental conditions). (c),(d) Lower panels: maximum of BLS intensity as a function of input laser wavelength, for $h$ and $v$ input, respectively. Upper panels: Reflected optical intensity (PD I), shown for comparison. The $x$ axis is detuning from the resonant wavelength of the $h$ polarized mode. For $v$ input measurements, this is set by the measured $h-v$ splitting $\lambda_{h-v}^{\text {eff }}$. For the optical modes $Q_{v} \approx 2 \times 10^{5}$ and $Q_{h} \approx 1 \times 10^{5}$ (dissipation rates $\kappa_{v} \approx 1 \mathrm{GHz}, \kappa_{h} \approx 2 \mathrm{GHz}$ ).

polarization for any input wavelength. The bottom panel shows a measured spectrum for $v$ polarized input. Here, there is only a Stokes peak (orange arrow), lower in frequency by the microwave drive. In the following, we demonstrate that this asymmetry between the Stokes or anti-Stokes signal [11,12], different for the two input polarization, follows from a selection rule in the BLS process. The linewidth of the BLS peak is limited by the $200 \mathrm{MHz}$ resolution of the etalon. We further note that when the magnetic field is reversed, the BLS is substantially reduced.

Figures 3(c) and 3(d) compare the BLS peak amplitude as a function of detuning of the input laser from the resonance to the reflectivity spectra. The BLS is enhanced when the $h(v)$ polarized input laser is resonant with the $h(v)$ polarized, $q=1$, WGM.
To explore the triple-resonance condition, the wavelength dependence of the BLS peak is measured as a function of the FMR frequency $\omega_{\mathrm{FMR}}$. This is shown in Fig. 4 for (b) $h$ and (c) $v$ input polarization. For $h(v)$ input, we only observe the Stokes (anti-Stokes) signal, and the color corresponds to the intensity of that signal. As the WGMs are sensitive to changes in sphere temperature with dissipated microwave power, the wavelength scans are aligned at the dip in reflected intensity (PD I), and are normalized to the peak value for that FMR frequency in order to highlight the mode structure. An example of the reflected intensities (PD I) for both input polarizations are shown for comparison in Fig. 4(a), these are independent of the FMR frequency.

In Figs. 4(b) and 4(c) there are two maxima in the efficiency of the BLS process. The first peak is independent of the FMR frequency and is aligned with the WGM of the input polarization. This corresponds to a cavity enhancement of the input light field. For small FMR frequencies there is a second peak whose wavelength is linear in the FMR frequency. For $h(v)$ polarized input, the black lines in Figs. 4(b) and 4(c) corresponding to $\omega_{v}-\omega_{\mathrm{FMR}}$ and $\omega_{h}+\omega_{\mathrm{FMR}}$, respectively, are in reasonable agreement with the data. Hence, the second peak corresponds to a cavity enhancement of output light field, shifted by the FMR frequency.

By tuning the FMR frequency to match the $h-v$ splitting, we achieve the triple-resonance condition. This scattering is between modes of different azimuthal mode indices,

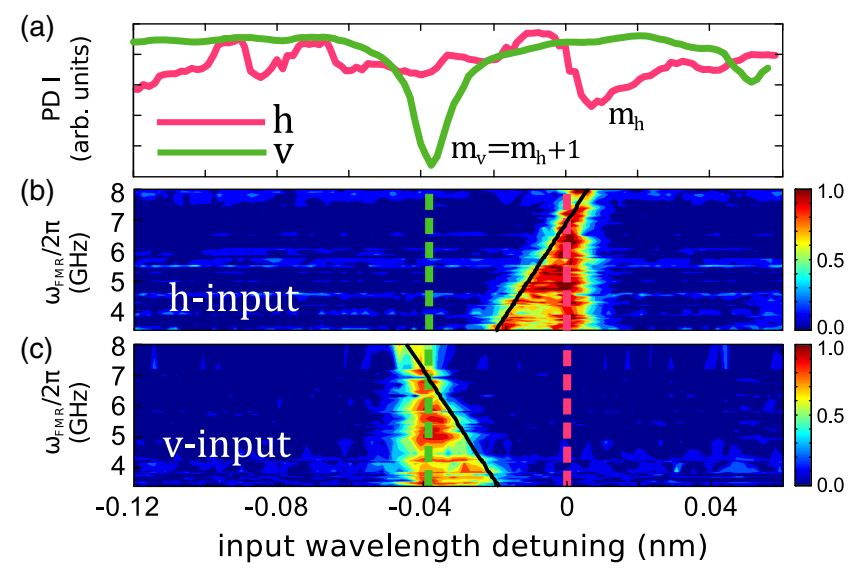

FIG. 4. BLS scattering amplitudes for different input linear polarizations ( $h$, pink, $v$, green). (a) Reflected intensity (PD I) for comparison. Azimuthal mode indices are labeled for clarity. The two curves are plotted on separate scales. (b) Color-plot of BLS intensity for $h$-input polarization as a function of input laser wavelength and FMR frequency. Each scan for fixed FMR frequency has been normalized to the peak amplitude for that scan. (c) As in (b), but for $v$-input polarization. Dashed lines in (b) and (c) indicate the resonant wavelengths for the two polarizations. The $x$ axis in all panels is in detuning from the resonant wavelength of the $h$ polarized mode. For $v$ input measurements, this is set by the measured $h-v$ splitting. 
$\Delta m= \pm 1$. In fact, this is consistent with our expectation, as, in the frame of the light propagating around the mode, the in-plane driven magnetization rotates with respect to the direction of propagation. This means that the magnetic mode has an effective wave vector, and azimuthal integration of the electromagnetic energy leads to a selection rule $m_{v}-m_{h}=1$ [28]. It is this required change in mode index that allows the triple-resonance condition to be achieved for reasonable magnetic field strengths, as the FSR is approximately equal to the $h-v$ splitting so that the two modes with $m_{v}-m_{h}=1$ are closely spaced in frequency. This is in contrast to previous work [11,12], which has suggested $\Delta m=0$, requiring substantially higher magnetic fields. We also note that in scattering the radial index $q$ is unchanged, $\Delta q=0$.

Furthermore, we can see that the Stokes or anti-Stokes asymmetry persists even detuned from the triple-resonance condition. This indicates that the asymmetry is not governed simply by the optical density of states. In fact, the selection rule $m_{v}-m_{h}=1$ means that the interaction Hamiltonian for the magnon mode $\hat{b}$ and two optical modes $\hat{a}_{h}, \hat{a}_{v}$, reduces to two terms [28], corresponding to the observed Stokes or anti-Stokes asymmetry, selected by the input polarization:

$$
\hat{H}_{\text {int }}=\hbar G\left(\hat{b} \hat{a}_{v}^{\dagger} \hat{a}_{h}+\hat{b}^{\dagger} \hat{a}_{h}^{\dagger} \hat{a}_{v}\right) .
$$

Hence, the scattering process is nonreciprocal due to the wave vector matching around the WGM and azimuthal dependence of the magneto-optical coupling. From the known strength of the Faraday effect in YIG, we calculate the single-photon coupling rate $G=1 \mathrm{~Hz}$ [28].

We can compare the measured data to a simple analytical model based on these three modes [28]. The amplitude of the scattered field as a function of the detuning from the triple-resonance condition $\omega_{\mathrm{FMR}}-\omega_{v}+\omega_{L}$ and of the $h$-polarized input frequency $\omega_{h}-\omega_{L}$ is

$$
\left|\left\langle\hat{a}_{v, \text { out }}\right\rangle\right|^{2}=\frac{4 G^{2}\left|\bar{a}_{h, \text { in }}\right|^{2}\left|\bar{b}_{\text {in }}\right|^{2} \kappa_{v} \kappa_{h} / \kappa_{\mathrm{FMR}}}{\left[\frac{\kappa_{h}^{2}}{4}+\left(\omega_{h}-\omega_{L}\right)^{2}\right]\left[\frac{\kappa_{v}^{2}}{4}+\left(\omega_{\mathrm{FMR}}-\omega_{v}+\omega_{L}\right)^{2}\right]} .
$$

This is the product of two Lorentzians, corresponding to resonant enhancement of the input and output fields, respectively. All the parameters are known from independent measurements, so that we can plot this expression in Fig. 5(b), with excellent agreement with the data plotted alongside [Fig. 5(a)].

Finally, we plot the maximum BLS amplitude for each FMR frequency in Fig. 5(c). The variation in the data is due to changes in the microwave power transmitted to the YIG sphere at different frequencies. The red line is the expected value given by Eq. (2), vertically scaled to match the data, with good agreement in the general trend.

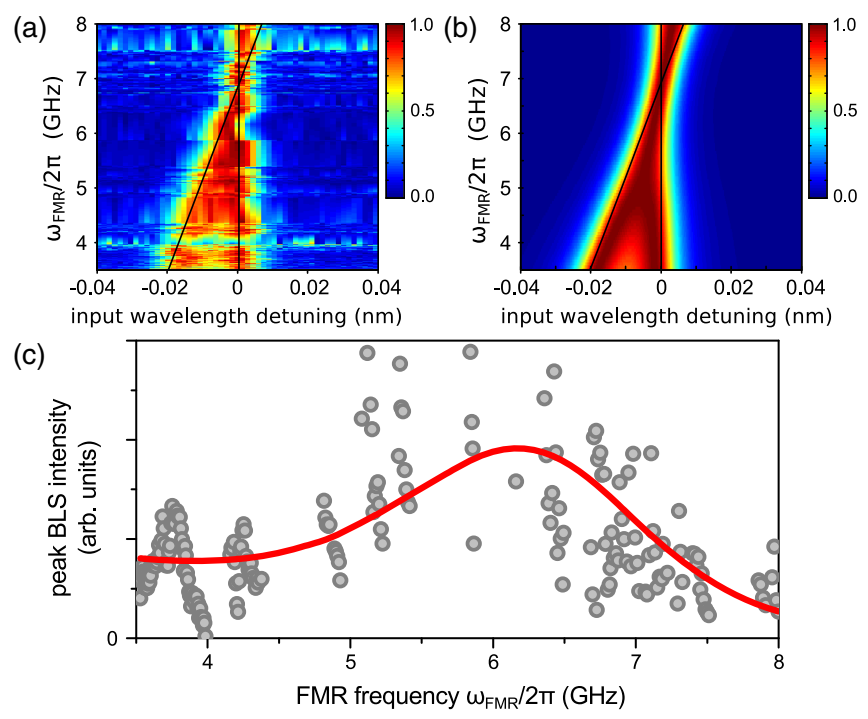

FIG. 5. Comparison between (a) experiment and (b) theory of BLS intensity as a function of input wavelength detuning and FMR frequency for $h$ input polarization. The black lines are the wavelengths corresponding to $\omega_{h}$ and $\omega_{v}-\omega_{\mathrm{FMR}}$. Both the experimental data and model are normalized to the peak value at each FMR frequency to allow better comparison of the mode structure. (c) Peak BLS efficiency as a function of FMR frequency. The red line is the expected trend given by the maximum of Eq. (2) for fixed $\omega_{\mathrm{FMR}}$.

To summarize, we have demonstrated the tuning of a cavity magneto-optical system to a triple-resonance condition for enhanced Brillouin light scattering. A selection rule $\Delta m= \pm 1$ in the azimuthal index of the optical mode arises due to wave vector matching around the optical path of the WGM. Because of the conservation of total angular momentum, a change in the optical orbital angular momentum of $\Delta m= \pm 1$ results in the annihilation or creation of one magnon, and up- or down-conversion of the light, respectively. The modes closest to energy-matching conditions have $m_{v}-m_{h}=1$, and, hence, the polarization of the input laser selects either a Stokes or anti-Stokes frequency conversion. Since the asymmetry of the BLS arises from a selection rule, a strong asymmetry can also be observed away from cavity resonance. This mechanism has similarities to BLS between two optical modes in optomechanics [29]. We further note that nontransverse components of the optical modes [30] are not included in our model, and are therefore not needed to explain the asymmetry in the BLS [11,12].

Asymmetries in magnon BLS have been reported previously due to other mechanisms. Localization of surface magnon modes with a given chirality [31] is not relevant here, as we study the uniform magnetic mode, and spinspin correlations between different components introduced by the demagnetizing field are only relevant in a thin film geometry [32]. It is possible that interference between the first- (Faraday) and second-order (Voigt) magneto-optical 
effects [16,33] may result in minor corrections to the differing amplitudes.

Although the single-photon coupling rate is significantly smaller than the linewidths of the optical and magnetic modes, the scaling of the coupling with the magnetic mode volume suggests that interesting regimes could be achieved with smaller devices. Optimizing the $Q$-factor mode volume ratio [34] could be achieved with photonic-crystal defect cavities [35], or plasmonic resonances, which have been shown to enhance static magneto-optical parameters [36-38]. In addition, for the current system, cooling to low temperatures [39] should improve the $Q$ factors of all modes significantly. This may open up the possibility of spontaneous Brillouin cooling [29] or lasing [40] of the magnetic mode in this system.

This project was partly funded by EPSRC under EP/ M50693X/1. A. J. F. is supported by ERC Grant No. 648613 and a Hitachi Research Fellowship. A. N. holds a University Research Fellowship from the Royal Society and acknowledges support from the Winton Programme for the Physics of Sustainability.

*jh877@cam.ac.uk

[1] C. A. Regal and K. W. Lehnert, From cavity electromechanics to cavity optomechanics, J. Phys. Conf. Ser. 264, 012025 (2011).

[2] M.H. Devoret and R.J. Schoelkopf, Superconducting circuits for quantuminformation: An outlook, Science 339, 1169 (2013).

[3] H. J. Kimble, The quantum internet, Nature (London) 453, 1023 (2008).

[4] J. Bochmann, A. Vainsencher, D. D. Awschalom, and A. N. Cleland, Nanomechanical coupling between microwave and optical photons, Nat. Phys. 9, 712 (2013).

[5] R. W. Andrews, R. W. Peterson, T. P. Purdy, K. Cicak, R. W. Simmonds, C. A. Regal, and K. W. Lehnert, Bidirectional and efficient conversion between microwave and optical light, Nat. Phys. 10, 321 (2014).

[6] T. Bagci, A. Simonsen, S. Schmid, L. G. Villanueva, E. Zeuthen, J. Appel, J. M. Taylor, A. Sørensen, K. Usami, A. Schliesser, and E. S. Polzik, Optical detection of radio waves through a nanomechanical transducer, Nature (London) 507, 81 (2014).

[7] K. C. Balram, M. I. Davanço, J. D. Song, and K. Srinivasan, Coherent coupling between radiofrequency, optical and acoustic waves in piezo-optomechanical circuits, Nat. Photonics 10, 346 (2016).

[8] D. V. Strekalov, A. A. Savchenkov, A. B. Matsko, and N. $\mathrm{Yu}$, Efficient upconversion of subterahertz radiation in a high-Q whispering gallery resonator, Opt. Lett. 34, 713 (2009).

[9] L. Chen, Q. Xu, M. G. Wood, and R. M. Reano, Hybrid silicon and lithium niobate electro-optical ring modulator, Optica 1, 112 (2014).
[10] A. Rueda, F. Sedlmeir, M. C. Collodo, U. Vogl, B. Stiller, G. Schunk, D. V. Strekalov, C. Marquardt, J. M. Fink, O. Painter, G. Leuchs, and H. G. L. Schwefel, Efficient microwave to optical photon conversion: an electro-optical realization, Optica 3, 597 (2016).

[11] A. Osada, R. Hisatomi, A. Noguchi, Y. Tabuchi, R. Yamazaki, K. Usami, M. Sadgrove, R. Yalla, M. Nomura, and Y. Nakamura, Cavity Optomagnonics with Spin-Orbit Coupled Photons, Phys. Rev. Lett. 116, 223601 (2016).

[12] X. Zhang, N. Zhu, C.-L. Zou, and H. X. Tang, Optomagnonic whispering gallery microresonators, arXiv:1510.03545.

[13] S. O. Demokritov, B. Hillebrands, and A. N. Slavin, Brillouin light scattering studies of confined spin waves: linear and nonlinear confinement, Phys. Rep. 348, 441 (2001).

[14] J. A. Haigh, S. Langenfeld, N. J. Lambert, J. J. Baumberg, A. J. Ramsay, A. Nunnenkamp, and A. J. Ferguson, Magneto-optical coupling in whispering-gallery-mode resonators, Phys. Rev. A 92, 063845 (2015).

[15] N. Brahms and D. M. Stamper-Kurn, Spin optodynamics analog of cavity optomechanics, Phys. Rev. A 82, 041804 (2010).

[16] H. le Gall and J. P. Jamet, Theory of the elastic and inelastic scattering of light by magnetic crystals. I. First-Order processes, Phys. Status Solidi B 46, 467 (1971).

[17] L. Deych, C. Meriles, and V. Menon, Resonant enhancement of magneto-optical polarization conversion in microdisk resonators, Appl. Phys. Lett. 99, 241107 (2011).

[18] T. Liu, X. Zhang, H. X. Tang, and M. E. Flatté, Optomagnonics in magnetic solids, Phys. Rev. B 94, 060405 (2016).

[19] S. V. Kusminskiy, H. X. Tang, and F. Marquardt, Coupled Spin-Light dynamics in cavity optomagnonics, arXiv: 1604.07053.

[20] M. L. Gorodetsky and V. S. Ilchenko, Optical microsphere resonators: optimal coupling to high-Q whispering-gallery modes, J. Opt. Soc. Am. B 16, 147 (1999).

[21] M. L. Gorodetsky and A. E. Fomin, Geometrical theory of whispering-gallery modes, IEEE J. Selected Topics Quant. Electronics 12, 33 (2006).

[22] I. Breunig, B. Sturman, F. Sedlmeir, H. G. L. Schwefel, and K. Buse, Whispering gallery modes at the rim of an axisymmetric optical resonator: Analytical versus numerical description and comparison with experiment, Opt. Express 21, 30683 (2013).

[23] These modes are often labeled quasitransverse magnetic $($ TM- $h$ ) and quasitransverse electric (TE- $v$ ) [11,12].

[24] G. Schunk, J. U. Fürst, M. Förtsch, D. V. Strekalov, U. Vogl, F. Sedlmeir, H. G. L. Schwefel, G. Leuchs, and C. Marquardt, Identifying modes of large whispering-gallery mode resonators from the spectrum and emission pattern, Opt. Express 22, 30795 (2014).

[25] P. C. Fletcher and R. O. Bell, Ferrimagnetic resonance modes in spheres, J. Appl. Phys. 30, 687 (1959).

[26] R. Engel-Herbert and T. Hesjedal, Calculation of the magnetic stray field of a uniaxial magnetic domain, J. Appl. Phys. 97, 074504 (2005).

[27] V. Kambersky and C. E. Patton, Spin-wave relaxation and phenomenological damping in ferromagnetic resonance, Phys. Rev. B 11, 2668 (1975). 
[28] See Supplemental Material at http://link.aps.org/ supplemental/10.1103/PhysRevLett.117.133602 for the derivations of Eqs. (1) and (2).

[29] G. Bahl, M. Tomes, F. Marquardt, and T. Carmon, Observation of spontaneous Brillouin cooling, Nat. Phys. 8, 203 (2012).

[30] C. Junge, D. O'Shea, J. Volz, and A. Rauschenbeutel, Strong Coupling between Single Atoms and Nontransversal Photons, Phys. Rev. Lett. 110, 213604 (2013).

[31] P. Grünberg and F. Metawe, Light Scattering from Bulk and Surface Spin Waves in EuO, Phys. Rev. Lett. 39, 1561 (1977).

[32] R. E. Camley, P. Grünberg, and C. M. Mayr, Stokes-antiStokes asymmetry in Brillouin scattering from magnons in thin ferromagnetic films, Phys. Rev. B 26, 2609 (1982).

[33] W. Wettling, M. G. Cottam, and J. R. Sandercock, The relation between one-magnon light scattering and the complex magneto-optic effects in YIG, J. Phys. C 8, 211 (1975).

[34] Y. Akahane, T. Asano, B.-S. Song, and S. Noda, High-Q photonic nanocavity in a two-dimensional photonic crystal, Nature (London) 425, 944 (2003).

[35] T. Yoshie, A. Scherer, J. Hendrickson, G. Khitrova, H. M. Gibbs, G. Rupper, C. Ell, O. B. Shchekin, and D. G. Deppe,
Vacuum Rabi splitting with a single quantum dot in a photonic crystal nanocavity, Nature (London) 432, 200 (2004).

[36] V. I. Safarov, V. A. Kosobukin, C. Hermann, G. Lampel, J. Peretti, and C. Marlière, Magneto-optical Effects Enhanced by Surface Plasmons in Metallic Multilayer Films, Phys. Rev. Lett. 73, 3584 (1994).

[37] P. K. Jain, Y. Xiao, R. Walsworth, and A. E. Cohen, Surface plasmon resonance enhanced magneto-optics (supremo): Faraday rotation enhancement in gold-coated iron oxide nanocrystals, Nano Lett. 9, 1644 (2009).

[38] V. V. Temnov, G. Armelles, U. Woggon, D. Guzatov, A. Cebollada, A. Garcia-Martin, J.-M. Garcia-Martin, T. Thomay, A. Leitenstorfer, and R. Bratschitsch, Active magneto-plasmonics in hybrid metal-ferromagnet structures, Nat. Photonics 4, 107 (2010).

[39] F. Treussart, V. S. Ilchenko, J.-F. Roch, J. Hare, V. LefèvreSeguin, J.-M. Raimond, and S. Haroche, Evidence for intrinsic Kerr bistability of high-Q microsphere resonators in superfluid helium, Eur. Phys. J. D 1, 235 (1998).

[40] S. M. Spillane, T. J. Kippenberg, and K. J. Vahala, Ultralowthreshold Raman laser using a spherical dielectric microcavity, Nature (London) 415, 621 (2002). 